\title{
Thrust stand based on a single point load cell for impulse measurements from plasma thrusters
}

\author{
Cite as: Rev. Sci. Instrum. 91, 023308 (2020); doi: 10.1063/1.5127189 \\ Submitted: 10 September 2019 Accepted: 12 January 2020 • \\ Published Online: 4 February 2020
}

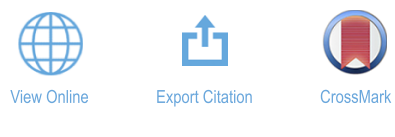

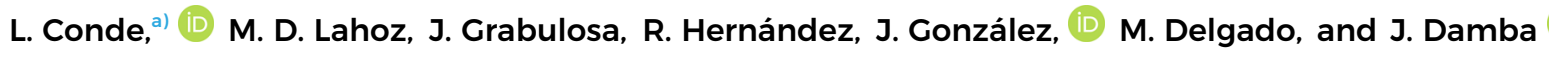

\begin{abstract}
AFFILIATIONS
Department of Applied Physics, Escuela Técnica Superior de Ingeniería Aeronáutica y del Espacio, Universidad Politécnica de Madrid, 28040 Madrid, Spain
\end{abstract}

a) Author to whom correspondence should be addressed: luis.conde@upm.es

\begin{abstract}
We introduce a simple thrust stand for the direct measurement of the millinewton impulses or thrusts delivered by small thrusters intended for in-space electric propulsion. The thruster under test, with a weight below $1.5 \mathrm{~kg}$, is disposed on a horizontal platform and its impulse is measured as an overweight by using a strain gauge cell physically protected from the ambient plasma and vacuum conditions. This system provides ten thrust readings per second with noise peak to peak amplitudes of $0.10-0.18 \mathrm{mN}$. The calibration procedures to verify its dynamic response to time dependent thrusts in the range of $0-15 \mathrm{mN}$ using control weights as well as its minimum thrust sensitivity $\delta T_{s}=0.3 \mathrm{mN}$ are discussed. Additionally, its simple conception permits a plain data reduction and analysis of steady state and low frequency thrust transients. This thrust stand was employed under low pressure and plasma ambient conditions to measure the steady impulses delivered by the Alternative Low Power Hybrid Ion Engine (ALPHIE) of $0.4-4.0 \mathrm{mN}$ with absolute errors $\Delta T= \pm 0.3 \mathrm{mN}$. Finally, the experimental results show that a control electric voltage governs the ALPHIE thruster throttle.
\end{abstract}

Published under license by AIP Publishing. https://doi.org/10.1063/1.5127189

\section{INTRODUCTION}

Electric propulsion (EP) systems for space propulsion are currently used for long-term maneuvering, station keeping, and endof-life disposal of satellites. They use physical processes in which electricity is involved to increase the exhaust velocity $v_{e x}$ of a gas stream and/or a plasma flow to values greater than those delivered by conventional chemical thrusters.

The EP systems accelerate particles to velocities $v_{e x} \gtrsim 10 \mathrm{~km} / \mathrm{s}$ to reach high specific impulse values $I_{s p}=v_{e x} / g_{o}$ where $g_{o}$ is the standard Earth's acceleration. However, the reduced electric power available on board of spacecrafts limits the propellant mass flow rate $\dot{M}$ that can be accelerated and, therefore, their actual momentum thrust $T=\dot{M} v_{e x}$ levels. Hence, electric thrusters are characterized by low thrusts and high specific impulses. ${ }^{1}$

The EP commercial systems available for telecommunication satellites deliver 50-200 millinewtons $(\mathrm{mN})$ with power consumption in the kilowatt range. In the opposite limit, impulses of $1-100$ micronewtons $(\mu \mathrm{N})$ are obtained by very low power systems such as electrospray or colloid thrusters intended for microsatellite technology. The intermediate range of thrust is essential for the orbital maneuvers of small satellites $(100-700 \mathrm{~kg})$ of the constellations intended for planetary internet coverage. They require typical thrusts in the range $0.1-50 \mathrm{mN}$, with electric power consumption roughly below $500 \mathrm{~W}$.

Direct and precise measurement of the delivered thrust range is essential to determine EP systems' performance. Retarded field ion energy analyzers allow us to measure the ion velocity distribution function and, therefore, the mean ion exhaust speed $v_{e x}$. Then, the determination of the specific impulse is independent of the thrust range. However, specific measurement techniques are required in accordance with EP system impulse levels. The thrust stands are complex and, in addition to measuring very small forces, they must operate at low pressure and, in many cases, also in a plasma that is an electrically active medium. This can affect the thrust stand performance. ${ }^{6}$

Pendulum and torsional balances are preferred for the micronewton impulse range of $1-100 \mu \mathrm{N}$ as they can provide precise measurements of thrust. Different configurations have been tested and discussed in comprehensive reviews. Basically, they have pendulum schemes or require a torsional force spring and/or actuator balanced with a damping mechanism to produce overdamped 
motion of the arm where the thruster under test is installed. This requisite limits the temporal response of pendulum and microbalance designs, which is not crucial since the thruster impulse changes have a slower time scale.

In addition to pendulum configurations, ${ }^{6-10}$ strain load cells and piezoelectric transducers with a better dynamic response can be used $^{11}$ for the millinewton range. However, their operation in vacuum and under ambient plasma conditions can be an issue since this physical medium is also chemically reactive. ${ }^{6}$ Free electrons and/or ions can be collected by plasma-exposed electrical connections, leading to stray currents. These can give rise to false readings or the damage of the electrical system. Additionally, adhesives employed to fix the strain load cell to the beam can be subjected first to outgassing and also to deteriorate under ion bombardment. These attract an ion current since metallic parts are usually electrically connected to the grounded chamber walls of the vacuum tank.

This paper follows the basic recommendations of Ref. 7 to characterize a thrust stand which provides impulse readings in one dimension. It is based on a single point strain gauge load cell insulated from the ambient plasma. Its simple operation and calibration procedures can be adapted to different microthrusters with typical weights below $1.5 \mathrm{~kg}$ delivering impulses in the $0-15 \mathrm{mN}$ range. As we shall see, the response of this system has been tested under low neutral gas pressures and also under realistic ambient plasma conditions using the Alternative Low Power Hybrid Ion Engine (ALPHIE) plasma thruster. ${ }^{12-15}$

The characteristics of the thrust stand are discussed in Sec. II and its calibration tests and performance are in Sec. III. The determination of impulses under realistic conditions with our ALPHIE plasma thruster is discussed in Sec. IV. The thrust stand allows a simple data reduction and analysis that are discussed in Sec. V. Finally, we end with some concluding remarks.

\section{THE THRUST STAND}

The plasma thrust stand of the scheme in Fig. 1 was disposed over a fixed horizontal aluminum platform of $2 \mathrm{~cm}$ thickness inside the cylindrical vacuum tank Arges of $2 \mathrm{~m}$ length and $80 \mathrm{~cm}$ in diameter. The baseline neutral gas pressure was of $1.6 \times 10^{-6} \mathrm{mbar}$ of argon or xenon that raises up to $1.0-10.0 \times 10^{-5}$ mbar for gas injections rates in the range $Q=0.3-1.0$ standard cubic centimeters per minute $(\mathrm{sccm})$. These are the propellant neutral gas injection rates required for the operation of the ALPHIE plasma thruster employed later for the impulse measurements under realistic conditions.

The thruster under test is installed on the upper surface of a horizontal aluminum platform of dimensions $19.5 \mathrm{~cm} \times 19.5 \mathrm{~cm}$ and $3 \mathrm{~mm}$ thickness shown in Fig. 1. Its impulse axis points along the vertical direction as shown in the photograph of Fig. 2. All electrical connections and gas pipes of the thruster were fixed to this platform using kapton tape to avoid their eventual uncontrolled displacements.

The plasma plume and the energized ion flow point upward and its subsequent expansion introduces important gradients in physical parameters, such as the charged particle densities or the electron temperature. The thrust stand is located at the back of the plasma plume, so only the low density background plasma interacts with its exposed surfaces. In the operation of the ALPHIE plasma thruster, the typical measured plasma densities are $n_{e} \sim 0.1-1.0 \times 10^{8} \mathrm{~cm}^{-3}$

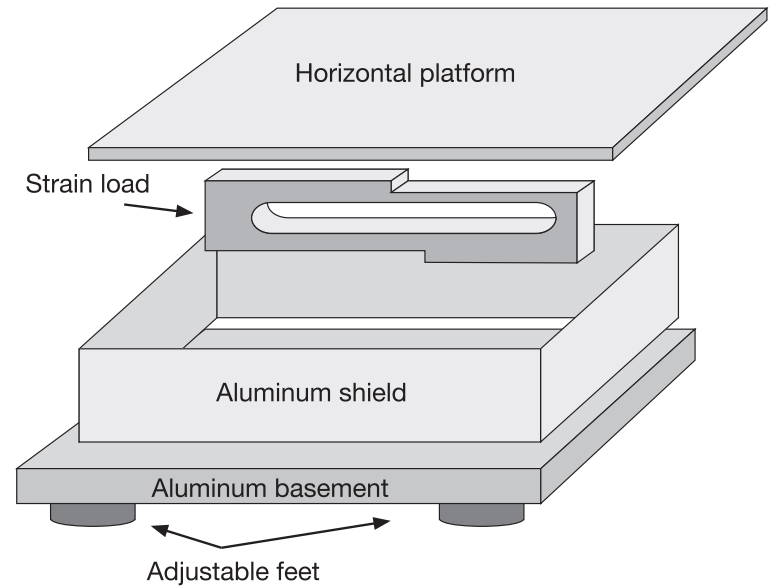

FIG. 1. The exploded view scheme of the thrust stand

and the electron temperatures are in the range $T_{e} \sim 1-2 \mathrm{eV}$. These values give typical Debye lengths of $\lambda_{D} \sim 1-7 \mathrm{~mm}$.

The upper face of a single point load cell (Vishay model 1015) is screwed to this platform and its lower side to a $0.5 \mathrm{~cm}$ thick aluminum base plate, which has four adjustable feet shown in Fig. 1. This allows us to control the horizontal alignment of the upper platform where the plasma thruster is deposited.

As the plasma is a chemically active medium, it can damage the sensitive strain gauge of the load cell and/or introduce stray currents in the measuring circuit. Therefore, an aluminum shield shown in Fig. 1 surrounds the strain gauge and beam that electrically insulates these sensitive elements. This metallic envelope was fixed

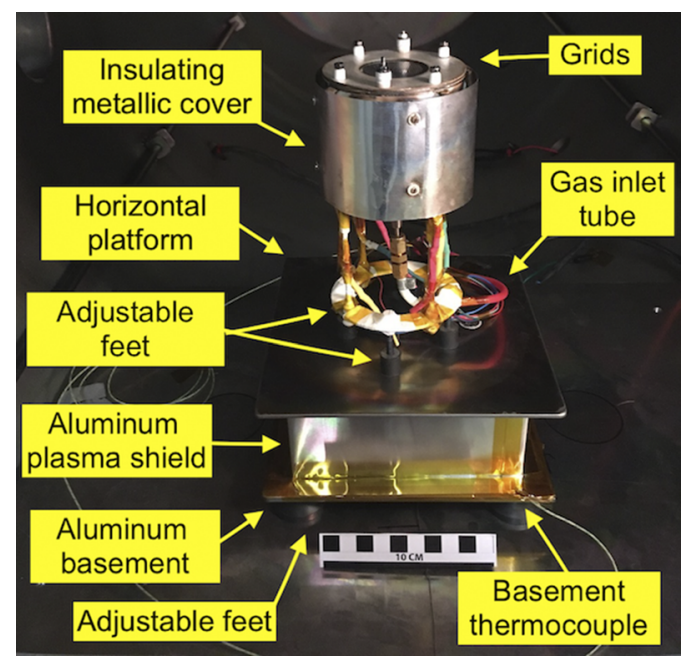

FIG. 2. The thrust stand setup and the ALPHIE plasma thruster with a reference scale in centimeters. The thruster external metallic cover is electrically unconnected to insulate its internal connections from the plasma. Its lower exposed metallic surfaces are protected with Teflon, and the vertical direction is adjusted by means of four adjustable feet. 
on the lower aluminum base. However, a thin groove of less than $\mathrm{d} \lesssim 1 \mathrm{~mm}$ width was left open between the metallic shield and the upper horizontal platform to allow for the small displacements of the strain load cell beam. Additionally, this groove permits to evacuate the air initially inside this enclosure in the gas pumping process.

All metallic parts of the thrust stand are electrically connected to the grounded plasma vacuum chamber walls. Since only the thin groove of width $\mathrm{d}<\lambda_{D}$ below the involved Debye lengths is left open, the thrust stand cavity enclosing the strain load cell constitutes an electrical Faraday cage. Therefore, all its sensitive elements are electrically insulated from the outside ambient plasma and ion bombardment, as well as from microwaves with typical wavelengths over $1 \mathrm{~mm}$.

Finally, since the thruster under test is vertically disposed, the delivered impulse is determined by its overweight measured by the strain gauge analogue signal. The later was fed into the Avery Weight-Tronix ZK830 controller which provides 10 weight readings per second that were transformed into thrust values using the standard Earth's acceleration.

In steady state conditions, the time average $\langle T\rangle$ of this signal provides the impulse and its mean standard deviation $\sigma_{T}$ is a quantitative measure of the mechanical noise for this impulse value. This is also the case for the null thrust signal $\langle T\rangle=0$ which has a $\sigma_{o}$ mean standard deviation. Thus, the absolute error of the $\langle T\rangle$ is the sum $\left(\Delta T=\sigma_{o}+\sigma_{T}\right)$ of the mechanical noise estimations for thrust and the null impulse levels.

\section{CHECKS AND CALIBRATIONS}

The proper operation of the thrust stand of Fig. 1 can be verified by simply placing weights equivalent to the expected impulses on the horizontal top platform where the thruster is installed. A set of metallic control weights were calibrated using the Avery WeightTronix ZK830 balance with a precision of $\Delta m= \pm 0.5 \mathrm{mg}$ for a maximum of $1.0 \mathrm{~kg}$ load.

Since $1 \mathrm{mN}$ impulse is equivalent to $0.102 \mathrm{~g}$, the thrusts equivalent to these control weights have an absolute error $\Delta m \times g_{o}= \pm 4.9$ $\times 10^{-3} \mathrm{mN}$. These were well below the $0.10-0.16 \mathrm{mN}$ mechanical noise peak to peak amplitudes observed in our thrust stand. Specifically, $\sigma_{T}= \pm 0.14 \mathrm{mN}$ in Fig. 3, which gives an absolute error $\Delta T$ $= \pm 0.3 \mathrm{mN}$ for the time average $\langle T\rangle$ of flat steps under the static load of control weights.

The thrust stand sensitivity is defined as the minimum impulse that can be realized over the $\pm 0.12-0.16 \mathrm{mN}$ amplitude noisy background. 'This sensitivity $\delta T_{s}=2 \sigma_{T} \simeq 0.3 \mathrm{mN}$ was estimated as two mean standard deviations of the maximum observed mechanical noise in Fig. 3.

The mechanical response of the impulse stand was also tested at low pressure $\left(10^{-6} \mathrm{mbar}\right)$ under a fixed load, similar to the weight of our ALPHIE plasma thruster $(1.1 \mathrm{~kg})$ to verify that no changes take place in the strain gauge load cell operating at low pressures or due to by outgassing processes of materials. The dispersion of these stationary readings was again of $\pm 0.12-0.16 \mathrm{mN}$ as in previous static tests at atmospheric pressure.

The final check was to verify the thrust stand performance again using controlled load weights under the ambient plasma produced by our ALPHIE thruster. In these tests, the vacuum tank pressure increases from $10^{-5} \mathrm{mbar}$ to $10^{-4} \mathrm{mbar}$ since the plasma is produced

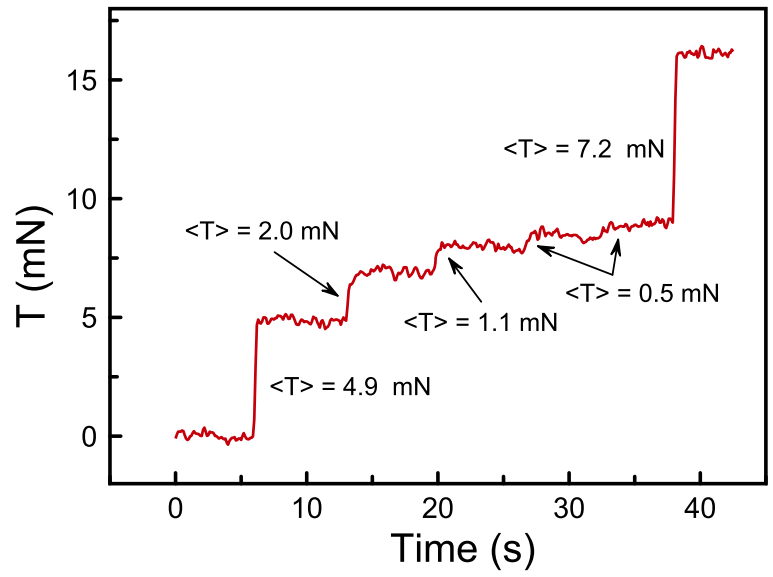

FIG. 3. Time dependent impulse readings when the control weights are successively deposited over the horizontal platform of Fig. 1. The time average of flat steps give $\langle T\rangle=0.0 \mathrm{mN}, 4.9 \mathrm{mN}, 2.0 \mathrm{mN}, 1.1 \mathrm{mN}, 0.5 \mathrm{mN}$, and $7.2 \mathrm{mN}$ with $\sigma_{0}$ $\simeq \sigma_{T}=0.14 \mathrm{mN}$ in all cases

by the fractional ionization of the thruster propellant gas (argon or xenon). The subsequent plasma plume expansion and recombination increments the background neutral gas pressure into the vacuum tank.

This test was intended to verify the effectiveness of the electric shielding of the strain gauge load cell discussed in Sec. II so that its readings remained unaffected by the ambient plasma conditions. Additionally, as some thruster elements are hot under their operational conditions, the temperature of the thrust stand was simultaneously monitored using a thermocouple. Since it was found within $\pm 3{ }^{\circ} \mathrm{C}$ of those of the vacuum chamber walls, we concluded that no heating effects were introduced by the plasma thruster operation.

Finally, impulse measurements were conducted by installing the ALPHIE thruster on the stand platform. The prototype makes use of an external control feed gas system and also laboratory power

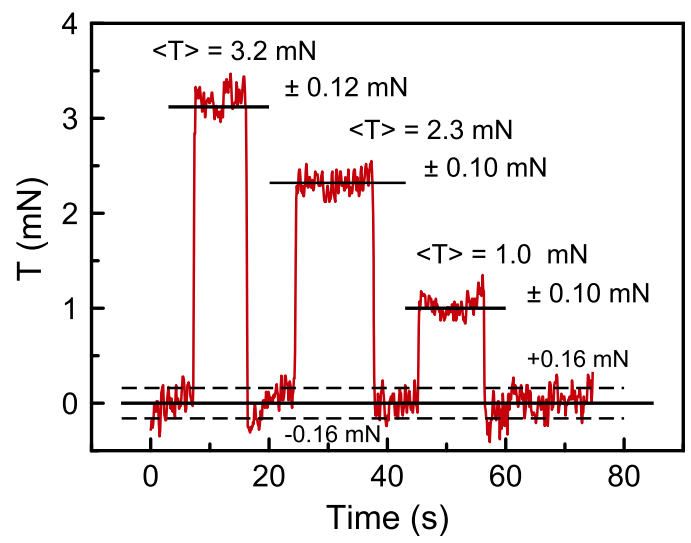

FIG. 4. Response in time to three successive test weights after impulse measurement testing with the plasma engine installed over the thrust stand. 


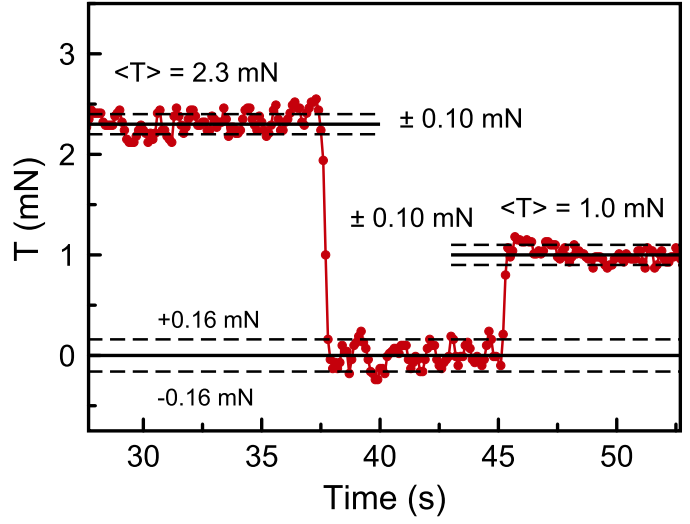

FIG. 5. Detail of temporal response in Fig. 4 when two different control weights are manually released and removed.

supplies connected through vacuum feedthroughs. This is a frequent situation in electric propulsion impulse testing.

In our case, electrical cables and the propellant gas supply tube can be simply fixed to the thrust stand platform using kapton tape. However, it was necessary to verify before thrust measurements if the expected impulses exerted by the thruster on the platform could eventually produce additional forces due to displacement of cables, propellant gas supply line, etc.

To exclude this possibility, additional tests were routinely made, before and after a series of impulse tests, using control weights hanging from a thin thread. These were manually deposited on and removed from the horizontal thrust stand platform in order to verify if a sudden impulse introduces an additional mechanical reaction.

The typical response to this dynamic check using three control weights after the impulse measurements is shown in Fig. 4. The solid lines indicate the averages $\langle T\rangle$ of stair steps that were found equal to impulses produced by the control weights. The dashed lines indicate that $\sigma_{T}= \pm 0.12 \mathrm{mN}$ and $\sigma_{T}= \pm 0.10 \mathrm{mN}$ for the thrust readings and the $\sigma_{o}= \pm 0.16 \mathrm{mN}$ is the error margin for the null impulse.

The steep rise and fall of $\sim 0.6 \mathrm{~s}$ in Fig. 5 confirms that thrust readings are produced by the three control weights and we found no additional mechanical responses. This final calibration process was conducted before and also after the experiments to asses any eventual changes during testing in the vacuum chamber.

\section{THE THRUST MEASUREMENT PROCESS}

The thrust stand was used to determine the impulses delivered by our ALPHIE plasma engine whose performances and operation have been previously discussed. ${ }^{12-15}$ Briefly, when the propellant neutral gas valve is open, a stationary mass flow rate $Q$ of argon or xenon gas is introduced into the plasma chamber of the thruster and the ion acceleration potential $V_{A C}$ between $450 \mathrm{~V}$ and $750 \mathrm{~V}$ is fixed. The typical thruster currents $I_{E}$ in the tests were in the range of 200-400 $\mathrm{mA}$ and the total electric power consumption of $200-325 \mathrm{~W} .{ }^{12}$

The only cathode is disposed in front of its two-grid acceleration system. When it is heated up to the thermionic emission temperature, a fraction of emitted electrons is accelerated by the voltage $V_{A C}$ through the open spaces of the grids. They move inward to the plasma chamber, where the propellant gas is injected, and are confined by the magnetic field configuration.

The unmagnetized ions resulting from the ionizing collisions of electrons with neutral gas atoms are accelerated outward by the $V_{A C}$ potential. In the stationary state, a counterflow is established through the grids of ionizing electrons from the cathode moving inward and supersonic ions that are accelerated along the opposite direction. Finally, the plasma beam exhaust is formed when additional electrons from the cathode neutralize the outgoing ion beam.

Contrary to conventional gridded ion engines, ALPHIE operation is governed by the electron emission from only one cathode. These electrons are responsible for both electric charge production and ion beam neutralization, similarly to Hall effect plasma thrusters. Figures 8 and 10 of Ref. 12 show the strong dependence of the thruster ion production rate on the electron emission from the cathode.

The ALPHIE thruster switches off when the electric current that heats the thermionic cathode turns off, so the ionizing electron flow extinguishes. This action interrupts the collisional ion production in the plasma chamber and hence the imparted momentum. Similarly, when the propellant gas valve is closed, keeping the cathode current constant, the ion production is also off, so the visible plasma plume disappears and the null impulse level is recovered. Therefore, the interaction of the cathode current with the magnetic field of the thruster does not introduce forces.

These specific features were used in the sequence of thrust measurements shown in Figs. 6 and 7. In both cases, the argon mass flow rate of $Q=0.6 \mathrm{sccm}$ and the acceleration potential $V_{A C}$ $=650 \mathrm{~V}$ were held constant. Figure 6 shows the time dependent thrust stand impulse signal as the cathode heating current was successively connected and interrupted three times using a manual electrical switch.

The arrows in Fig. 6 indicate points when the cathode was on and off, and solid lines show the average $\langle T\rangle=3.2 \mathrm{mN}$ with $\Delta T$ $= \pm 0.33 \mathrm{mN}$ absolute error. Dashed lines indicate the $\sigma_{T}= \pm 0.2 \mathrm{mN}$ value of thrust fluctuations and the $\sigma_{o}= \pm 0.13 \mathrm{mN}$ of the null thrust $\langle T\rangle=0$ noise level.

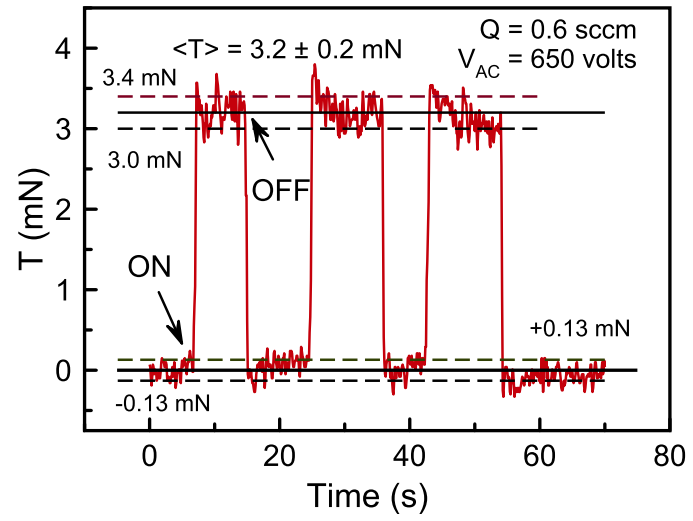

FIG. 6. The thrust signal as a function of time when the cathode of the plasma thruster is successively switched on and off three times. 


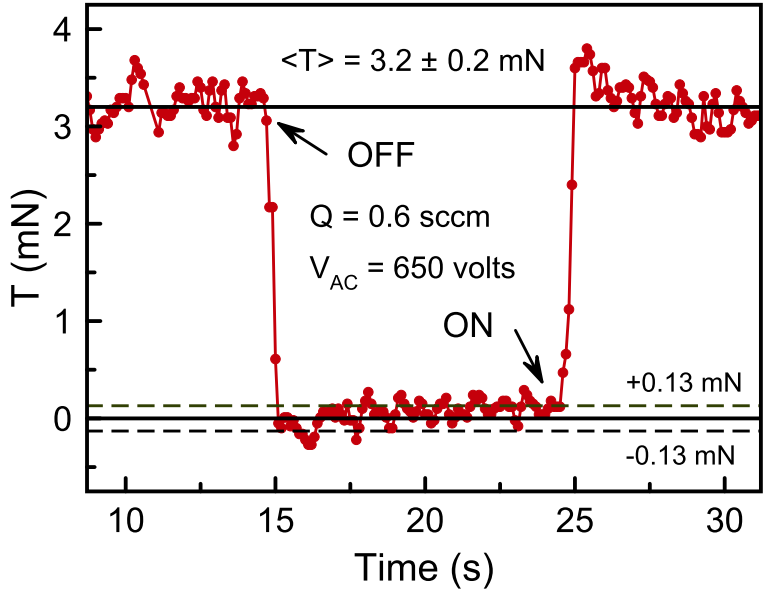

FIG. 7. Detail of the rise/fall response in time in Fig. (6) as the cathode is successively switched off and on where it is indicated by the arrows.

When the cathode heating was disconnected, no ions are produced and the momentum imparted by the $0.6 \mathrm{sccm}$ flow of neutral argon gas injected into the thruster plasma chamber is below the minimum $\delta T_{s} \sim 0.3 \mathrm{mN}$ sensitivity threshold of the thrust stand.

The thrust experienced a steep jump when the cathode heating current is switched on. The impulse fluctuations around the average $\langle T\rangle=3.2 \mathrm{mN}$ are essentially caused by oscillations in the ionizing electron flow rate. When the cathode heating current is turned off again, the null impulse level is recovered after a sharp drop of thrust.

The three thrust jumps in Fig. 6 show the electron emission on/off process, which was typically repeated between 5 and 15 times in the impulse measurements for fixed operational conditions of the ALPHIE thruster. The average calculated for each of the three

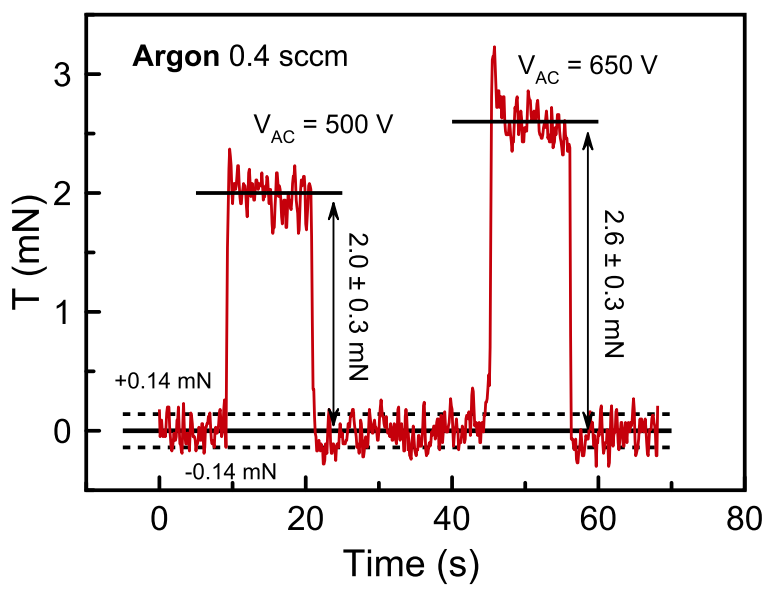

FIG. 8. Throttle produced by a $500 \mathrm{~V}$ and $650 \mathrm{~V}$ acceleration voltages. The solid lines indicate the $\langle T\rangle=2.0 \mathrm{mN}$ and $\langle T\rangle=2.6 \mathrm{mN}$ average thrust delivered and the $\langle T\rangle=0$ null impulse level. Dashed lines show the $\sigma_{0}= \pm 0.14 \mathrm{mN}$ null thrust noise level. jumps in this figure was $\langle T\rangle=3.2 \mathrm{mN}$ within the $\sigma_{T}= \pm 0.2 \mathrm{mN}$ dashed lines' margin. However, the impulse absolute error is $\Delta T$ $= \pm 0.33 \mathrm{mN}$, considering also the time fluctuations $\sigma_{o}= \pm 0.13 \mathrm{mN}$ of the null thrust signal.

The details of the temporal response of thrust to this ion production on/off process are shown in Fig. 7. Since 10 data are digitized per second, we can estimate the rising edge of the thrust signal as $\sim 0.6 \mathrm{~s}$, whereas the falling edge approximately takes $\sim 0.4 \mathrm{~s}$ from the $\langle T\rangle=3.2 \mathrm{mN}$ average down to the null thrust signal.

In Fig. 8 are compared the thrusts delivered for $V_{A C}=500 \mathrm{~V}$ and $V_{A C}=650 \mathrm{~V}$ acceleration voltages for the fixed mass flow rate of $0.4 \mathrm{sccm}$ of argon. The increment from $\langle T\rangle=2.0 \mathrm{mN}$ up to $\langle T\rangle$ $=2.6 \mathrm{mN}$ is achieved by increasing the acceleration potential from $V_{A C}$, while holding other ALPHIE operation parameters constant. This throttle capability of momentum thrust $T=\dot{m}_{i} v_{e x}$ is only caused by the electric field acceleration of ions up to supersonic speeds $v_{e x}$ and is a key performance of the ALPHIE plasma thruster.

\section{DATA REDUCTION}

The steady state impulse delivered by the plasma thruster can be determined as in Figs. 6 and 7 from the time average $\langle T\rangle$ of about 100 thrust readings or equivalently a $20 \mathrm{~s}$ duration impulse. As discussed in Sec. II, its absolute error is $\Delta T=\sigma_{T}+\sigma_{o}$ to account for the null impulse $\langle T\rangle=0$ error signal.

A quantitative measure of the repeatability of the thrust stand readings ${ }^{7}$ is the calculation of the mean standard deviation $\sigma_{m}$ of the average,

$$
\bar{T}=\sum_{i=1}^{N}\left\langle T_{i}\right\rangle,
$$

of a series of individual impulse jumps $\left\langle T_{i}\right\rangle$, as those in Fig. 6. The index $\sigma_{m}$ characterizes both the stability of delivered thrust and the repeatability of the impulse readings. We have found in practice that using typically 5-15 jumps in the impulse measurement process, the values of $\sigma_{m}$ are always below the estimate of the absolute error $\Delta T$ $=\sigma_{o}+\sigma_{T}>\sigma_{m}$. Therefore, $\bar{T}$ can be considered the thrust delivered and $\Delta T$ its absolute error for fixed operational conditions of the thruster. This was the case of Fig. 6 where $\bar{T}=3.2 \mathrm{mN}$ and $\Delta T$ $=0.33 \mathrm{mN}$.

In Figs. 6 and 7, the repeatability rate $\sigma_{m}$ was determined using the ALPHIE fast response to the on/off switching of the electrons source. However, a similar response can be obtained by switching on and off the gas leak valve that permit to extend present data analysis to other plasma thrusters different from the ALPHIE engine.

\section{CONCLUSIONS}

The simple thrust stand described in Sec. II makes use of a strain gauge cell electrically protected from the ambient plasma. ${ }^{6}$ Additionally, it can be used with other kinds of plasma thrusters of typical weights below $1.5 \mathrm{~kg}$.

The operation of the thruster under test in a vertical position makes the thrust stand, described in Sec. II, easy to operate. This feature permits the simple static and dynamic calibration procedures using control weights discussed in Sec. III. The strain load cells have a faster dynamic response than thrust stand designs based on 
damping and torsional spring schemes. However, its performance is below these more precise thrust balances designed for lower thrusts delivered by microthruster technology.

Figure 6 shows this thrust stand to provide reliable time dependent measurements in the range $0-15 \mathrm{mN}$ (Fig. 3) acquired at a sample rate of 10 readings per second. The peak to peak amplitude fluctuations of its time dependent impulse signal are of $\pm(0.10-0.16)$ $\mathrm{mN}$ which give a minimum $\delta T_{s}=0.3 \mathrm{mN}$ sensitivity. The simple impulse data reduction analysis discussed in Sec. $V$ gives typical absolute errors of $\Delta T \pm 0.3 \mathrm{mN}$.

This sensitivity and absolute error bounds have been confirmed under low pressure testing and realistic ambient plasma conditions and also in the measurements of impulse delivered by the ALPHIE thruster in Figs. 6-8. Additionally, the time response to calibrations and to the on/off switch of the ALPHIE plasma thruster can be estimated from Figs. 5 and 7 as a fraction of a second.

Future improvements will be a faster data sampling rate of the strain gauge load cell signal and to reduce the effect of ambient mechanical noise. A faster data acquisition rate of the thrust signal will be necessary to resolve the eventual transient thrust peaks observed in Figs. 6 and 8.

Finally, we have found that thrust measurements are rather sensitive to the specific ambient conditions of the laboratory, such as oscillations introduced by other equipment in the building and mechanical noise. Eliminating error sources requires careful test planning of and performing the systematic calibration tests of the thrust stand response discussed in Sec. III.

\section{ACKNOWLEDGMENTS}

The authors are grateful to the technical assistance of Mr. F. Sánchez and Mr. J. Damba to the Spanish FPI fellowship program.
This work was funded by Aernnova Aerospace S.A. and by the Ministry of Science, Innovation and Universities of Spain under Grant No. RT2018-094409-B-100.

\section{REFERENCES}

${ }^{1}$ S. Mazouffre, Plasma Sources. Sci. Technol. 25, 033002 (2016).

${ }^{2}$ E. Ahedo, Plasma Phys. Controlled Fusion 53, 124037 (2011).

${ }^{3}$ C. Charles, J. Phys. D: Appl. Phys. 42, 163001 (2009).

${ }^{4}$ D. M. Goebel and I. Katz, Fundamentals of Electric Propulsion. Ion and Hall Thrusters (John Wiley \& Sons, Hoboken, New Jersey, USA, 2008).

${ }^{5}$ M. Keidar, T. Zhuang, A. Shashurin, G. Teel, D. Chiu, J. Lukas, S. Haque, and L. Brieda, Plasma Phys. Controlled Fusion 57, 014005 (2015).

${ }^{6}$ S. Pottinger, D. Lamprou, A. Knoll, and V. Lappas, Rev. Sci. Instrum. 83, 033504 (2012).

${ }^{7}$ J. E. Polk, A. Pancotti, T. Haag, S. King, M. Walker, J. Blakely, and J. Ziemer, J. Propul. Power 33, 539 (2017).

${ }^{8} \mathrm{U}$. Kokal and M. Celik, in Proceedings of the 35th International Electric Propulsion Conference, Atlanta, Georgia USA, 2017, IEPC paper 2017-317.

${ }^{9}$ O. Neunzig, C. Drobny, and M. Tajmar, in Proceedings of the 35th International Electric Propulsion Conference, Atlanta, Georgia USA, 2017, IEPC paper 2017375.

${ }^{10}$ D. Frollani, M. Coletti, and S. Gabriel, Meas. Sci. Technol. 25, 065902 (2014).

${ }^{11}$ R. Stephen, K. Rajanna, V. Dhar, K. Kalyan Kumar, and S. Nagabushanam, Meas. Sci. Technol. 12, 1568 (2001).

${ }^{12}$ L. Conde, J. Domenech-Garret, J. Donoso, J. Damba, S. Tierno, E. AlamilloGamboa, and M. Castillo, Phys. Plasmas 24, 123514 (2017).

${ }^{13}$ L. Conde, J. L. Domenech-Garret, J. M. Donoso, E. Del Río, and M. A. Castillo, "Plasma accelerator with modulated thrust," US patent 10172227B2 (2019).

${ }^{14}$ L. Conde, J. L. Domenech-Garret, J. M. Donoso, E. Del Río, and M. A. Castillo, "Plasma accelerator with modulated thrust and space born vehicle with the same," European Patent EP3369294B1 (2015).

${ }^{15}$ J. Damba, P. Argente, P. Maldonado, A. Cervone, J. Domenech-Garret, and L. Conde, J. Phys.: Conf. Ser. 958, 012002 (2018). 